\title{
Strategy for Controlling the Level in a Dissolved Air Flotation Chamber
}

\author{
Rodrigo Almeida Galdino ${ }^{1}$, Alex Elton de Moura ${ }^{2,3}$, Valdemir Alexandre dos Santos ${ }^{1,3}$ and Leonie Asfora \\ Sarubbo $^{1,3^{*}}$ \\ 1. Center for Sciences and Technology, Catholic University of Pernambuco, Recife-PE 50.050-900, Brazil \\ 2. Center for Technology and Geosciences, Federal University of Pernambuco, Recife-PE 50.050-900, Brazil \\ 3. Center for Technology and Innovation Management, Recife-PE 50.050-900, Brazil
}

\begin{abstract}
The aim of the present study was to implant an efficient strategy for controlling the level of the effluent-oily foam interface in a DAF (dissolved air flotation) chamber of a pilot-scale prototype. DAF has been successfully used in the treatment of oily water, which is one of the main environmental problems in different industrial facilities. Along with important operational parameters, such as microbubble size and flow rate, the control strategy for the automation of a DAF chamber may be an important tool for increasing efficiency. Controlling the level was the strategy chosen to enhance the separation efficiency in a pilot-scale DAF prototype, with monitoring performed using a computational program in LabVIEW (laboratory virtual instrument engineering workbench). The findings demonstrate that it is possible to maintain the level of the fluid at a reference value established by the operator using the software program through the application of classic proportional integral derivative controllers. Using this control tool, the efficiency of water-oil separation in the pilot flotation chamber prototype was increased to nearly $98 \%$.
\end{abstract}

Key words: Dissolved air flotation, level control, LabVIEW, proportional integral derivative, liquid-liquid separation.

\section{Symbols Used}

$\begin{array}{ll}\overline{\mathrm{Q}}\left[\mathrm{m}^{3} \cdot \mathrm{s}^{-1}\right] & \text { volumetric flow rate } \\ \overline{\mathrm{H}}[\mathrm{m}] & \text { height } \\ \mathrm{T}[\mathrm{s}] & \text { time }\end{array}$

\section{Abbreviations}

$\begin{array}{ll}\text { DAF } & \text { dissolved air flotation } \\ \text { PID } & \text { proportional integral derivative } \\ \text { DAQ } & \text { data acquisition } \\ \text { LabVIEW } & \begin{array}{l}\text { laboratory virtual instrument engineering } \\ \text { workbench }\end{array}\end{array}$

\section{Introduction}

As environmental legislation has become increasingly rigorous, compliance requires more than merely removing the free-floating oil from oily water. It is also necessary to remove nearly the entirety of the oil in the form of emulsion [1]. The treatment process

\footnotetext{
*Corresponding author: Leonie Asfora Sarubbo, professor, research fields: liquid-liquid separation and biosurfactants. E-mail: leonie@unicap.br.
}

involves the following steps [2]: (1) Decantation for the removal of the free-floating oil whenever this portion is significant to avoid the greater consumption of chemical products in the following step; (2) Physicochemical treatment to break down the emulsion as well as to coagulate and flocculate the oil droplets; (3) Flotation for the separation of the oily phase flocculated from the treated water.

Separation through flotation occurs with the injection of air bubbles in the aqueous medium, followed by the adsorption of dispersed solid or liquid particles, which are carried to the surface of the medium. In most cases, these particles become hydrophobic due to the addition of the appropriate chemical products [3].

DAF (dissolved air flotation) is a separation process in which air bubbles produced by the depressurization of an aqueous current saturated with air at a pressure greater than the atmospheric pressure are used to capture the particles [1-3]. The separation of oil 
through flotation is considered the best option for the treatment of oily water containing either free-floating or emulsioned oil due to the low cost (compact equipment), operational ease and considerable efficiency, thereby ensuring compliance with environmental requirements and often allowing the reuse of the water [4].

Like every productive process, the equipment should be automated so that the system can be maintained in a steady state throughout its operation [5]. Considering the need to control the mixture of fluids and maintain the level of the foam-liquid interface in the flotation chamber, the development of strategies to meet such needs should be associated with the enhancement of the separation efficiency in the chamber [6]. It is possible to maintain the level of the liquid in a DAF chamber in accordance with previously established references values using process control systems and the development of controllers [7, 8]. Control systems are basically composed of measurement elements (sensors), logic control elements (controllers) and performance elements (valves). These elements are responsible for a set of measurement, comparison and calculation operations as well as the correction of deviations [9].

Among the different types of controllers, the PID (proportional integral derivative) is a control mechanism that calculates the error between a measured value and a desired value [10]. This type of controller is employed when a corrective action is needed to adjust a given process, thereby minimizing the error through a proportional action zeroed by the integral action and anticipated by the derivative action [11, 12]. This mechanism is based on the response of the mathematical model of the process to be controlled [13]. Adjustments to the control mechanism can be tested in a virtual environment with the aid of a graphic interface before being placed into practice. This can be done using the LabVIEW ${ }^{\circledR}$ (laboratory virtual instrument engineering workbench) from National Instruments. Using this graphic interface, the results of the control of the level in the DAF process can be visualized by testing the variables to be controlled in function of the response time of the controller [14].

This paper describes a control strategy based on the use of process control methods and the graphic interface of the LabVIEW program to evaluate the variation in the liquid level in a DAF chamber and maintain the level at a previously established reference. The influence of this type of control on the efficiency of the process is also evaluated.

\section{Materials and Methods}

\subsection{DAF Prototype}

The development of control strategies in a DAF chamber was performed using a prototype containing two connected chambers with walls made of transparent acrylic and supported by a steel framework. Fig. 1 shows the side view of the prototype used for the experiments before and after the implantation of the automation and control strategy. From left to right, Fig. 1 shows the oily water input duct (1), the influent flow is forced through one chamber with a static agitator (2), preliminarily mixing with the flow of a flocculation assistance substance in an auxiliary tank (3). The pre-flocculated mixture passes to a second chamber equipped with a mechanical paddle-type agitator (4) activated by an electric motor. With a satisfactory degree of blending, the water-oil mixture comes into contact with a flow of microbubbles that exit a sparger (5) in a preliminary flotation chamber (6). The mixture of the flocculated influent with microbubbles produces oily foam that floats due to the difference in density in comparison with the water and is gathered in a collector (7) as the foam overflows from the chamber. The water (largely free of oil) passes to a second flotation chamber (8) in which the interaction process is initiated again with a new flow of microbubbles.

The microbubbles enter the flotation chambers through distribution ducts (9). The treated water exits 


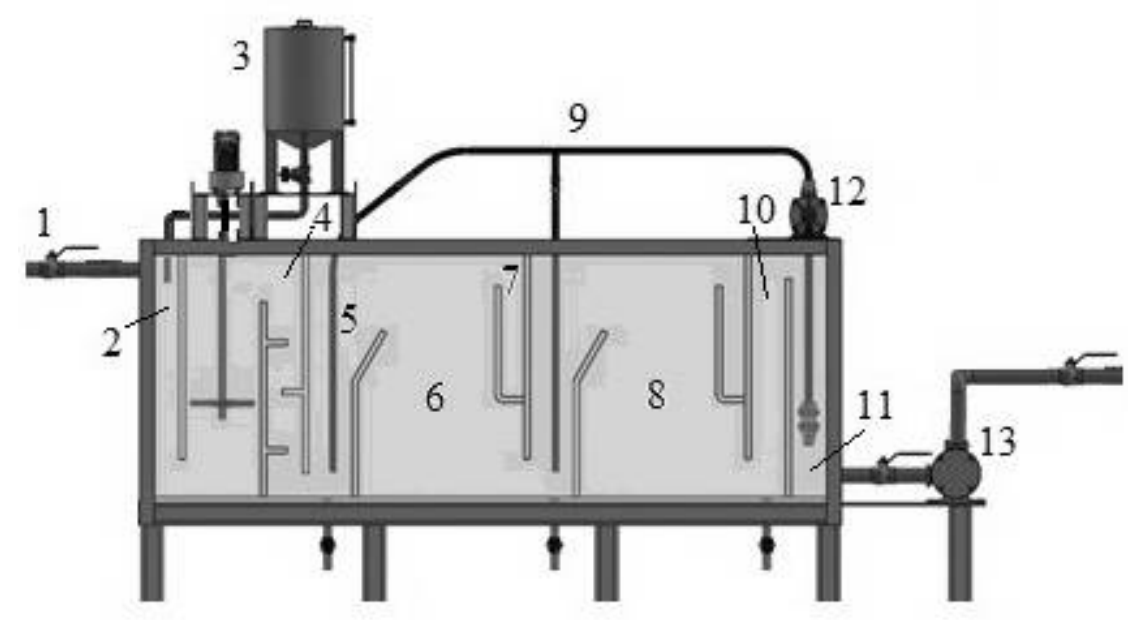

Fig. 1 Schematic showing layout of components of DAF protype.

the second flotation chamber and is forced through two connected chambers (10 and 11). In the second chamber, a centrifuge pump (12) recycles part of the water for the production of microbubbles. A duct at the base of this chamber (13) releases the treated water.

\subsection{Control Strategy}

Fig. 2 illustrates the level control strategy for implantation in the DAF prototype. Fig. 2 shows the following:

$\bar{Q}$ : Outflow through DAF prototype in permanent regimen;

$\bar{H}$ : Height of water column in DAF chamber of the prototype;

$q_{i}$ : Slight variation in input flow;

$q_{o}$ : Slight variation in output flow;

$H$ : Slight variation in height of water column $(\overline{\mathrm{H}})$.

The main hypothesis of this analysis is that a system linearized around the equilibrium positions (permanent regimen) produces $\overline{\mathrm{Q}}$ and $\overline{\mathrm{H}}$. Thus, the output flow in a permanent regimen is:

$$
Q=k \sqrt{H}
$$

where, $k$ is a proportionality constant.

The resistance $R_{T}$ of the outflow to the turbulent flow is

$$
R_{T}=\frac{d H}{d Q}
$$

Based on Eq. (1),

$$
\frac{d H}{d Q}=\frac{2 \cdot \sqrt{H}}{k}=\frac{2 H}{Q}
$$

Thus,

$$
R_{T}=\frac{2 \cdot H}{Q}
$$

In the liquid level system, the relationship for the restriction valve at the exit of the treated water is:

$$
R=\frac{h}{q_{o}}
$$

and the capacitance $C$ of the reservoir is defined as the variation in the amount of stored liquid necessary to cause a unit change in the height of the liquid column in the flotation chamber. Thus:

$$
C \cdot \frac{d h}{d t}=q_{i}-q_{o}
$$

Applying the Laplace transform to Eqs. (4) and (5):

$$
\begin{gathered}
R=\frac{H(s)}{Q_{o}} \Rightarrow Q_{o}=\frac{H(s)}{R} \\
C \cdot s \mathrm{H}(\mathrm{s})=Q_{i}(s)-Q_{o}(s)
\end{gathered}
$$$$
C \cdot s \mathrm{H}(\mathrm{s})=Q_{i}(s)-\frac{H(s)}{R} \Rightarrow
$$$$
\mathrm{H}(\mathrm{s})\left[C \cdot s+\frac{1}{R}\right]=Q_{i}(s)
$$ 


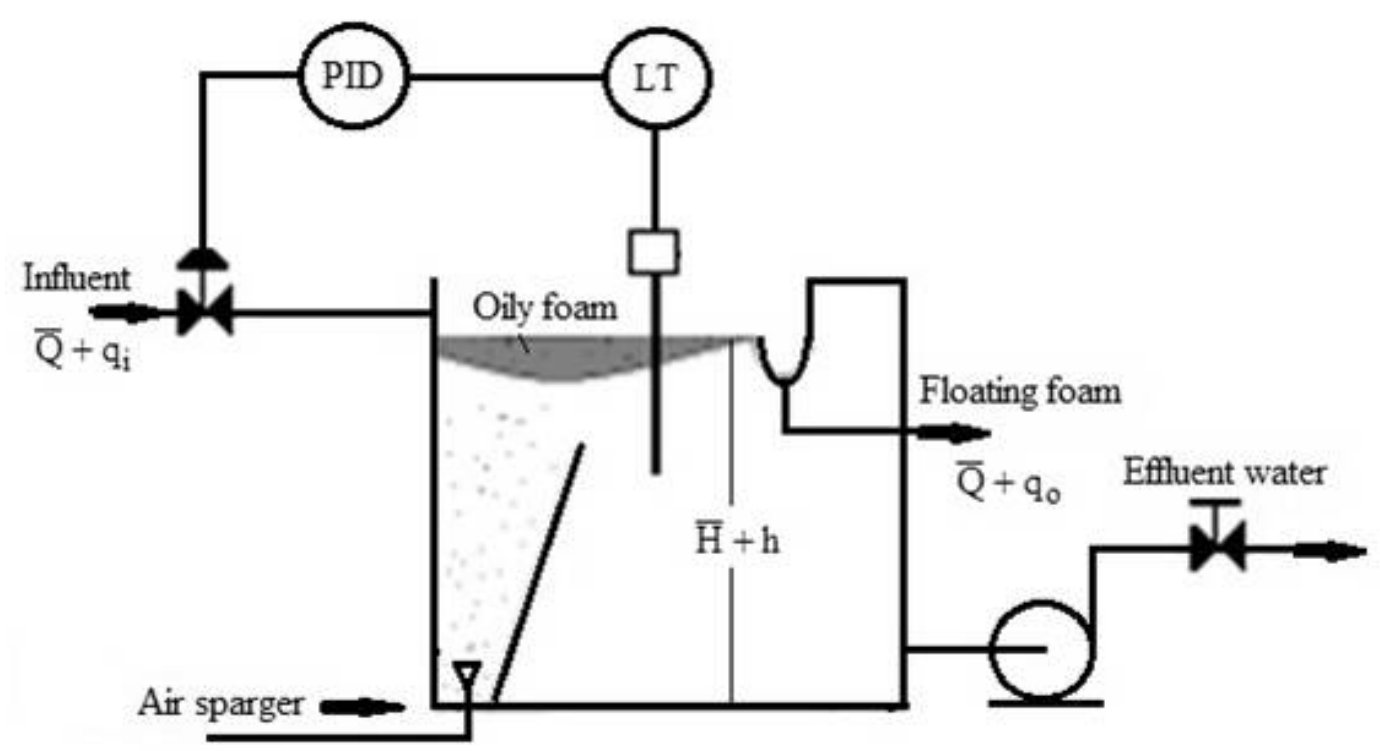

Fig. 2 Schematic used to model transference function of level control strategy in DAF prototype.

and the transference function is:

$$
\frac{H(s)}{Q_{i}(s)}=\frac{R}{R \cdot C \cdot s+1}
$$

\subsection{Control Software}

LabVIEW is a versatile tool for working in different environments, such as Windows and Linux, and can be used in simulation applications, data acquisition, laboratory automation and monitoring processes. This program allows working in process control with a high degree of reliability, differing from other languages by using its own programming and presentation code, denominated $\mathrm{G}$ (graphics) language [15].

The implementation of the PID control was performed in the LabVIEW environment using the virtual instruments necessary for the creation of the block diagram (Fig. 3), which are structures for programming and the flow of input and output information of the system [16]. Moreover, the main program and monitoring system were developed to control the level in the DAF unit.

\subsection{Data Acquisition System}

A data acquisition system made up of a DAQ/USB6008 board from National Instruments was used for the level control tests. This board allows the manipulation of analog and digital input and output signals using LabVIEW as the interface [17]. The module was connected to the interface board, which has electronic circuits used to measure changes in the level of the liquid in the DAF chamber and signal conditioning (Fig. 4).

A sensor and valve were used to detect and control the change in the level in the DAF chamber (Fig. 5). The LK3122 electronic level sensor determined the fluid level based on the capacitive measuring principle. The sensor measured the current level and generated an appropriate analog output signal. The analog output was configurable as an electrical current ranging from 4 to $20 \mathrm{~mA}$ or output tension ranging from 0 to $10 \mathrm{~V}$. An LR24SR valve served as the proportional operator; when the water in the flotation chamber reached a level considered to be the maximum, the valve progressively closed, thereby reducing the re-circulation flow in the DAF chamber to prevent overflow.

\section{Results and Discussion}

\subsection{PID Control}

A program with the PID function block was 


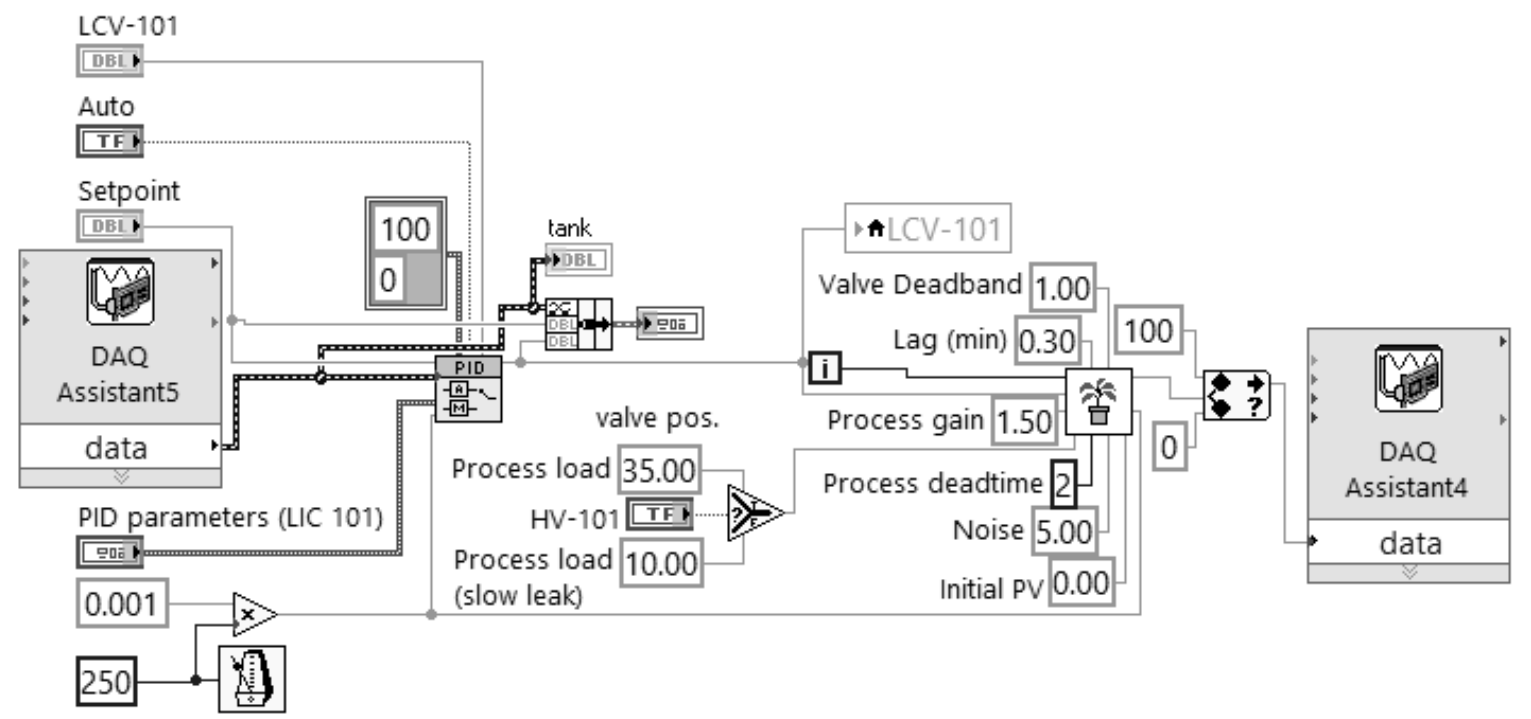

Fig. 3 Block diagram for level control.

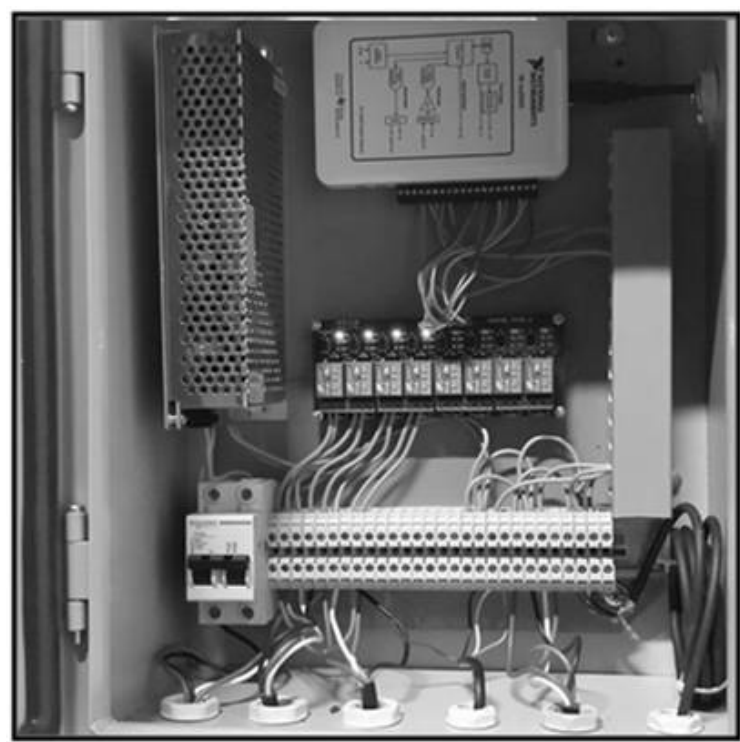

Fig. 4 Command board and data acquisition of control system.

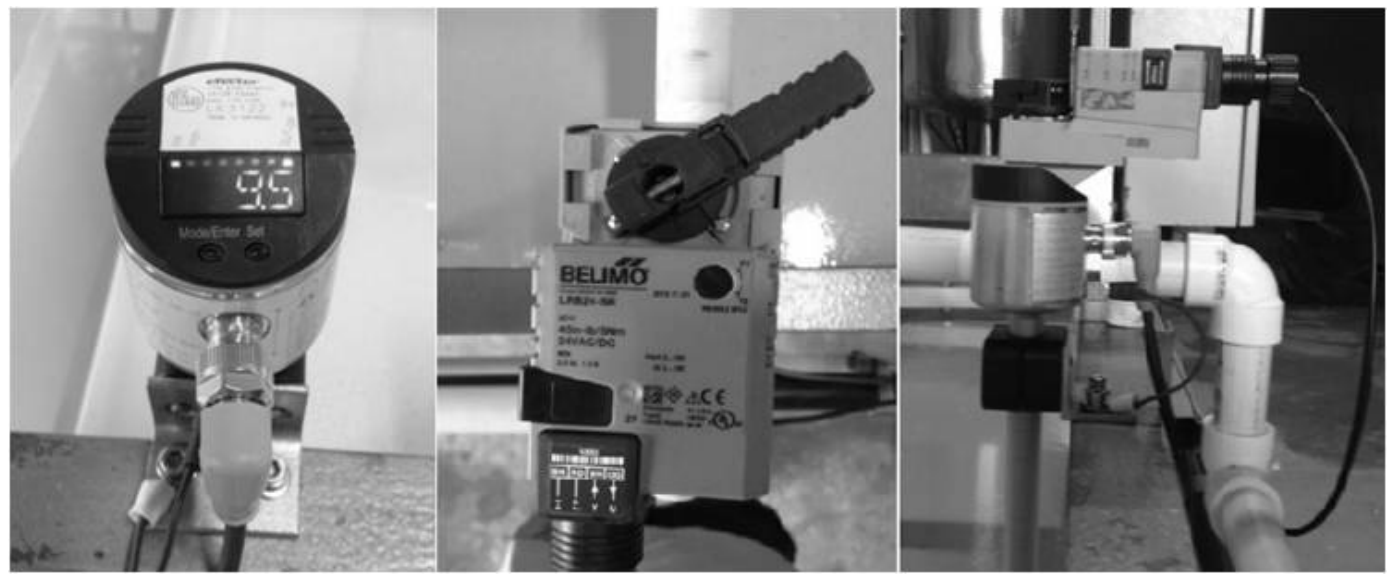

Fig. 5 Control elements (sensor and valve). 
developed in LabVIEW to control the level in the DAF chamber through the determination of proportional gain $(\mathrm{Kp})$, integral time (Ti) and derivative time (Td) (Fig. 6) are required to adjust the PID controller. Fluctuations or perturbations generated in the system have a user-defined set point as reference [18].

Ten centimeters were selected as the set point, which was considered the ideal level of the liquid in the DAF chamber. A response graph (Fig. 7) was created to evaluate the performance of the controller. This graph exhibits the occurrence of an overshoot, which is a peak value higher than the reference (set point) reached by the system [19]. The overshoot occurred in a 60 -second interval until stabilizing in a permanent regimen and equaling the process value to the set point.

\subsection{Water-Oil Separation Efficiency (Visual Response)}

Experiments were conducted in two situations to quantify the effects of the control strategy on water-oil separation efficiency. The tests were performed with a synthetic effluent in which the water was artificially contaminated by blending it with lubricating oil

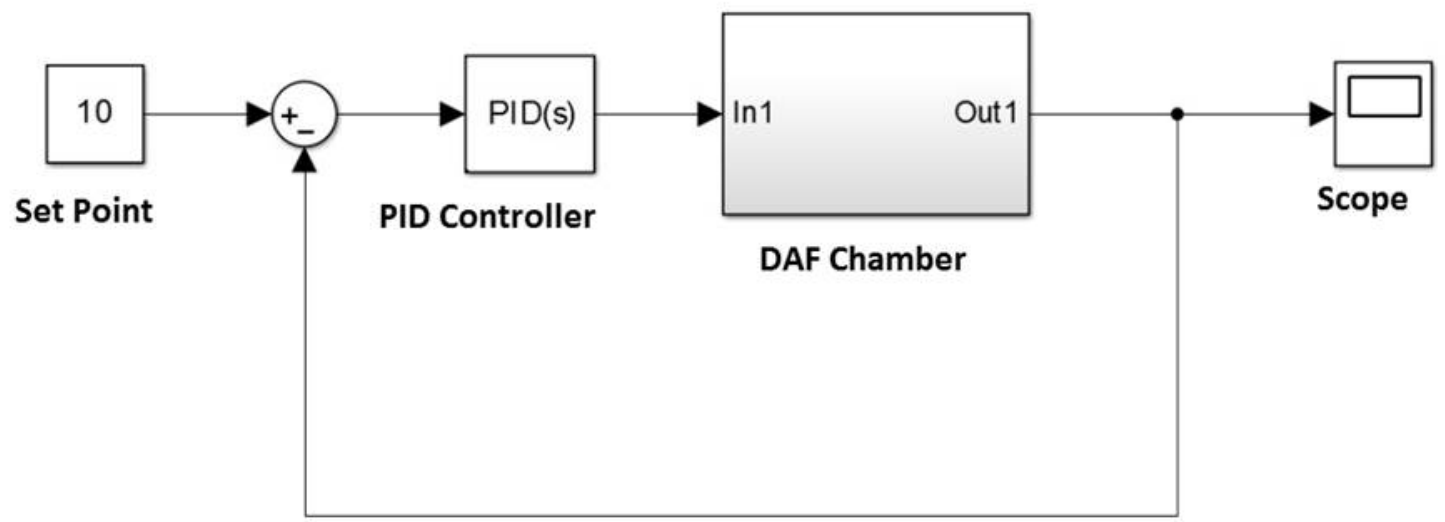

Fig. 6 Main program developed for level control.

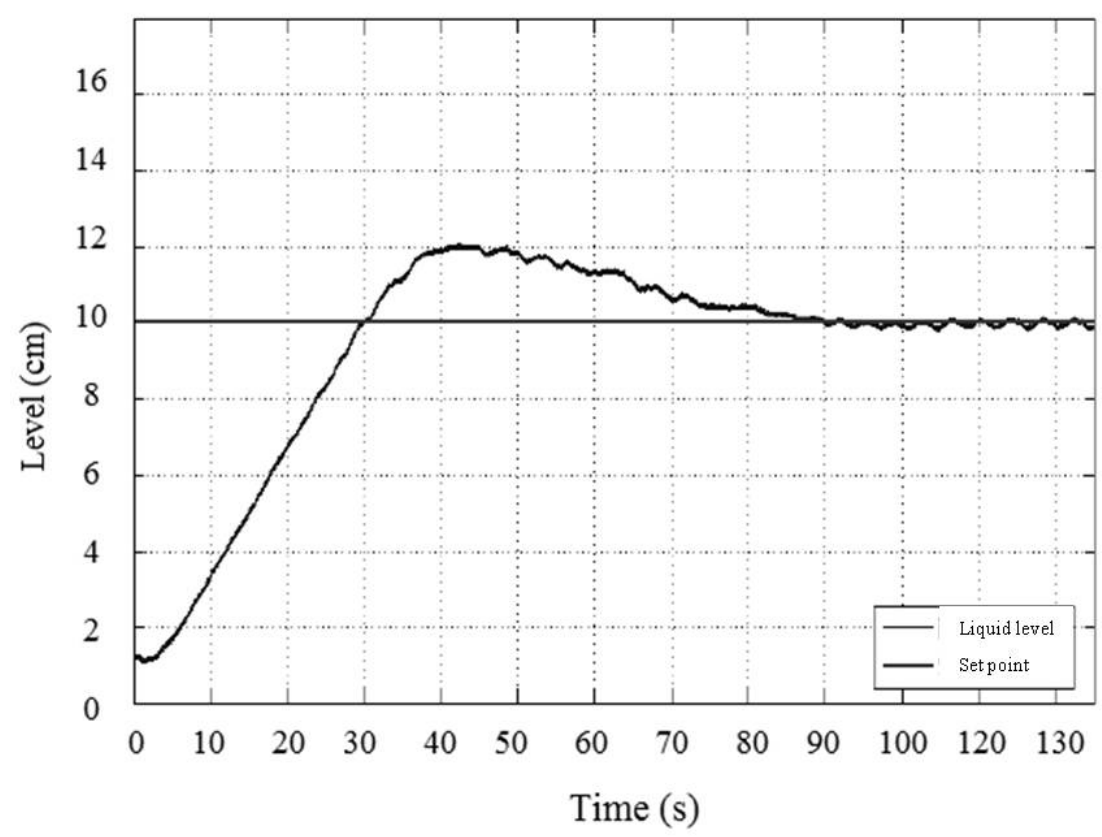

Fig. 7 Response graph of PID control. 
(Series 500, API SJ 20W-50). In the first flotation chamber, with the control strategy employed, the level sensor controlled the height of the liquid column through an increase or reduction in the opening of a control valve at the entrance of the prototype, thereby increasing or diminishing the return of part of the influent to the water-oil mixing tank. In the flotation chamber, the interaction between the flow of the synthetic influent and microbubbles could be adjusted to result in a chamber with a greater density of oily foam.

The water treated in the first chamber passed to the second chamber through an opening in the lower portion between the two chambers. Visual observation regarding the free surface of the second chamber revealed effect duplicity in both chambers due to the control strategy employed in the first chamber. The interaction between the flow of the synthetic influent containing residual oil in the second chamber and a second flow of microbubbles also gave rise to a thick layer of oily foam in the second DAF chamber. In the treated water chamber (last compartment of the DAF prototype), two centrifuge pumps directed the effluent. Part of this water was re-circulated for the production of microbubbles and part was re-circulated to receive artificial contamination and be re-fed into the DAF prototype.

Fig. 8 is a photograph of the free surface of the flotation chamber of the pilot DAF prototype during preliminary tests without the implantation of the automation and control system, on which a supernatant very poor in oily foam is evident. Fig. 9 is a photograph of the free surface of the flotation chamber after the implantation of the control system, on which a dense layer of oily foam is evident. Both tests were conducted under the same conditions, differentiated only by the use of the control strategy.

\subsection{Response Surface Analysis}

Fig. 10 shows the three-dimensional response surface graph of the DAF prototype for the separation of water and oil without the use of the level control strategy described by Rocha e Silva et al. [4]. This graph shows the separation efficiency the two flotation chambers in function of the independent variables: the ratio between the air and water flow for the production of microbubbles $\left(\mathrm{X}_{1}\right)$ and the ratio between the effluent to be treated and the treated water for the production of microbubbles $\left(\mathrm{X}_{2}\right)$. Simultaneous changes in $\mathrm{X}_{1}$ and $\mathrm{X}_{2}$ produced maximum separation efficiency, demonstrating an optimum point for the operational conditions of the prototype.

Fig. 11 shows the three-dimensional response surface graph of the DAF prototype after the implementation of the level control strategy using the same independent variables. Controlling the level in the first chamber increased the total separation efficiency of the system from approximately $78 \%$ to $98 \%$.

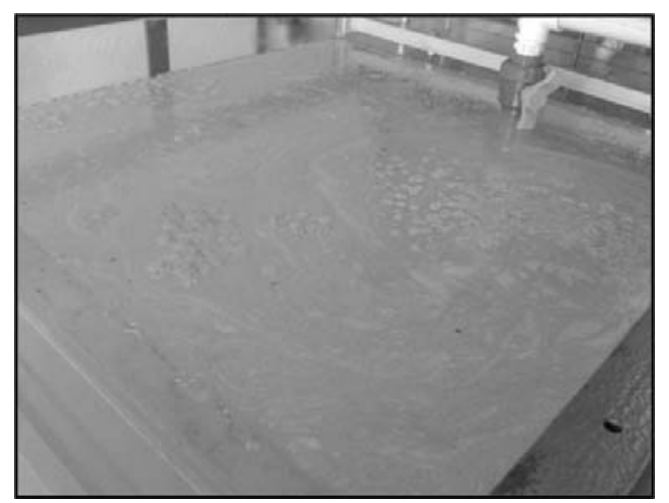

Fig. 8 Photograph of oily foam formed on free surface of flotation chamber prior to implementation of level control strategy.

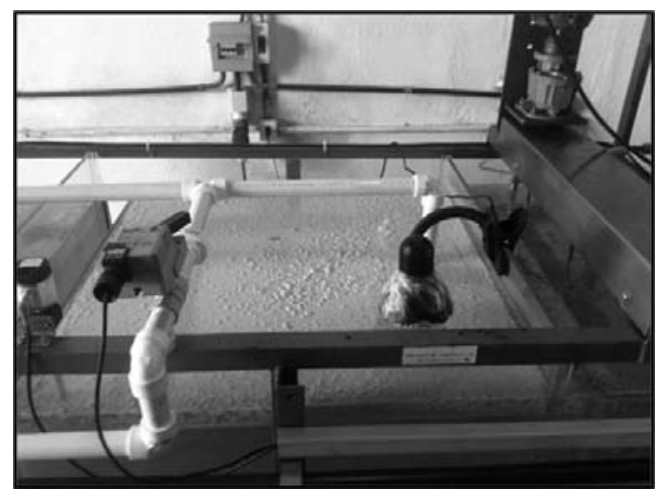

Fig. 9 Photograph of oily foam formed on free surface of flotation chamber after implementation of level control strategy. 


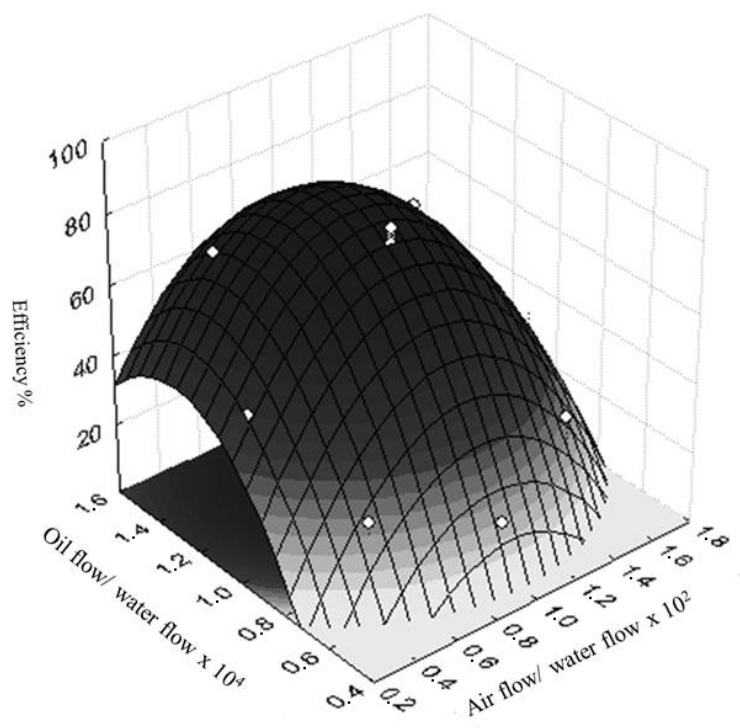

Fig. 10 3D surface response graph of separation efficiency of DAF prototype prior to implementation of level control strategy.

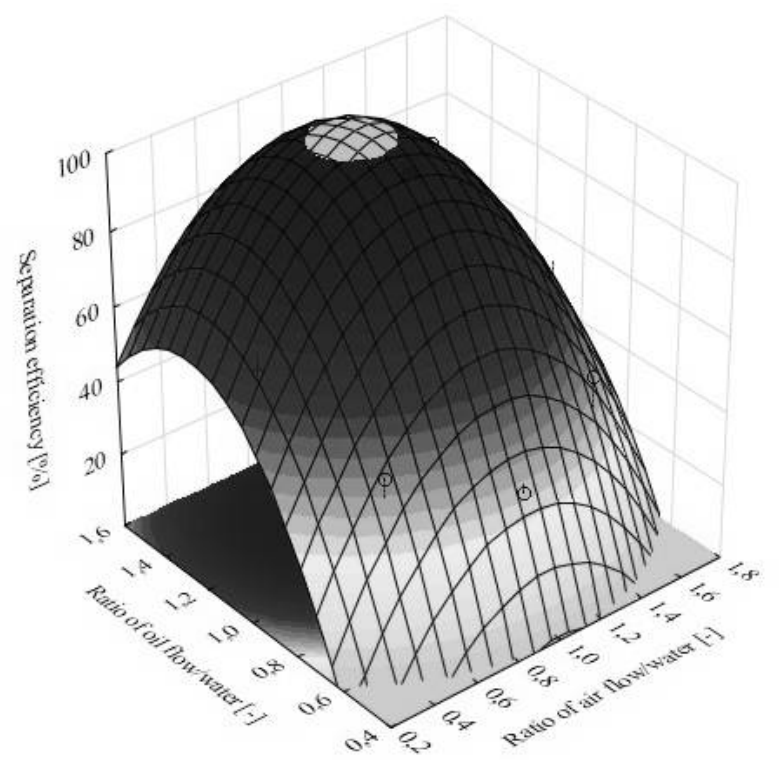

Fig. 11 3D surface response graph of separation efficiency of DAF prototype after implementation of level control strategy.

\section{Conclusions}

Quantitative and qualitative comparisons of the water-oil separation efficiency in a flotation chamber of the DAF prototype demonstrated the essential contribution of the level control strategy to the success of this method. The dissolved air flotation process involves a large number of important parameters, such as operational variables and the properties of the fluids. Among the operational variables, the ratios among the water, air and oil flows should be taken into account whenever the aim is to enhance the separation efficiency.

\section{Acknowledgments}

Funding for this study was provided by the State of Pernambuco Foundation for the Assistance to Science and Technology (FACEPE), the Research and Development Program of the Brazilian National Electrical Energy Agency (ANEEL), the National Council for Scientific and Technological Development (CNPq) and the Federal Agency for the Support and Evaluation of Graduate Education (CAPES). The authors are grateful to the laboratories of the Center for Science and Technology of the Catholic University of Pernambuco and the Center for Technology and Innovation Management (CGTI), Brazil.

\section{References}

[1] Oliveira, C., and Rubio, J. 2012. "A Short Overview of the Formation of Aerated Flocs and Their Applications in Solid/Liquid Separation by Flotation." Minerals Eng. 39: 124-32.

[2] Haarhoff, J., and James K. E. 2013. "Adapting Dissolved Air Flotation for the Clarification of Seawater." Desalination 311: 90-4.

[3] Zhu, H. Y., and Ming, Z. 2014. "Air Flotation Method in the Treatment of Oily Wastewater Application of Its Progress." Adv. Mat. Res. 971: 2044-7.

[4] Rocha e Silva, F. C. P., da Rocha e Silva, N. C. P., de Moura, A. E., Galdino, R. A., Luna, J. M., Rufino, R. D., and Sarubbo, L. A. 2015. "Effect of Biosurfactant Addition in a Pilot Scale Dissolved Air Flotation System." Sep. Sci. Technol. 50: 618-25.

[5] Zhao, L., Peng, T., Han, H., Cao, W., Lou, Y., and Xie, X. 2014. "Fault Condition Recognition Based on Multi-scale Co-occurrence Matrix for Copper Flotation Process." In Proceedings of the 19th World Congress, 7091-7.

[6] Behin, J., and Bahrami, S. 2012. "Modeling an Industrial Dissolved Air Flotation Tank Used for Separating Oil from Wastewater." Chem. Eng. Processing: Process Intensification 59: 1-8.

[7] Nikačević, N. M., Huesman, A. E., Van den Hof, P. M., and Stankiewicz, A. I. 2012. "Opportunities and 
Challenges for Process Control in Process Intensification." Chem. Eng. Processing: Process Intensification 52: 1-15.

[8] Yang, Y., Zhu, Q., Maasoumy, M., and Sangiovanni-Vincentelli, A. 2012. "Development of Building Automation and Control Systems." IEEE Design \& Test of Computers 29: 45-55.

[9] Romagnoli, J. A., and Palazoglu, A. 2012. Introduction to Process Control. New York: CRC Press, Taylor \& Francis Group.

[10] Segovia, V. R., Hägglund, T., and Åström, K. J. 2014. "Measurement Noise Filtering for PID Controllers." $J$. Process Control 24: 299-313.

[11] Paul, P. M., Dey, C., and Mudi, R. K. 2014. "An Online Dynamic Set Point Weighting Scheme for PID Controller." In Proceedings of the Students' Technology Symposium, 188-92.

[12] Åström, K. J., and Wittenmark, B. 2013. Adaptive Control. New York: Courier Dover Publications.

[13] Formentin, S., Campi, M. C., and Savaresi, S. M. 2014. "Virtual Reference Feedback Tuning for Industrial PID Controllers". In Proceedings of the 19th International Federation of Automatic Control World Congress, $11275-80$.
[14] Jensen, M. B., and Tallman, D. E. A. 2013. "LabVIEW-based Virtual Instrument for Simulation and Analysis of SECM Approach Curves." J. Solid State Electrochem. 17: 2999-3003.

[15] Chattopadhyay, S., Roy, G., and Panda, M. 2011. "Simple Design of a PID Controller and Tuning of Its Parameters Using LabVIEW Software." Sensors \& Transducers 129: 1726-5479.

[16] Naveenkumar, R., and Prasad. K. 2013. “Low Cost Data Acquisition and Control Using Arduino Prototyping Platform and LabVIEW." International Journal of Science and Research 2 (2): 366-9.

[17] Chouder, A., Silvestre, S., Taghezouit, B., and Karatepe, E. 2013. "Monitoring, Modelling and Simulation of PV Systems Using LabVIEW." Solar Energy 91: 337-49.

[18] Kienle, A., Sommer, S., and Nguyen, H. N. 2014. "Auto-tuning of Multivariable PI/PID Controllers Using Iterative Feedback Tuning: Design Examples." In Proceedings of the 24th European Symposium on Computer Aided Process Engineering, 721-6.

[19] Wali, W. A., Cullen, J. D., Bennett, S., and Al Shamma'a, A. I. 2013. "Intelligent PID Controller for Real Time Automation of Microwave Biodiesel Reactor." Int. J. Comp. Inf. Technol. 2: 809-14. 